\title{
TOWARDS THE UNIFICATION OF INCONSISTENCY HANDLING MECHANISMS*
}

\begin{abstract}
It is shown that the (flat) consequence relations defined from the Rescher-Manor Mechanism (that is: in terms of maximal consistent subsets of the premises) are all inconsistency-adaptive logics combined with a specific interpretation schema for the premises. Each of the adaptive logics is obtained by applying a suitable adaptive strategy to the paraconsistent logic CLuN.

This result provides all those consequence relations with a (dynamic) proof theory and with a static (as well as a dynamic) semantics.
\end{abstract}

\section{Aim of this paper}

Many inconsistency handling mechanisms are inspired by the idea that inconsistent sets of sentences may be divided into maximally consistent subsets - henceforth MCS, and that what 'follows' from the inconsistent set may be defined, along more or less complex lines, in terms of the classical consequences of these subsets. To the best of my knowledge, the idea was first applied in [20]. The application was a specific one (mainly counterfactuals). Nevertheless, many ideas that became popular later were already present. For example, Rescher clearly distinguishes between the recognition

\footnotetext{
* Research for this paper was supported by subventions from the Fund for Scientific Research - Flanders, and indirectly by the INTAS-RFBR contract 95-365. I am indebted to Kristof De Clercq and Liza Verhoeven for locating mistakes in an earlier draft.
} 
and the resolution of inconsistencies, and attributes these tasks to different mechanisms - see [20, p. 37]: "And while the recognition of ambiguity does fall within the province of logic, its resolution is inevitably an extralogical matter."

The full-blown machinery, this time phrased as an inconsistency handling mechanism, was articulated by Nicholas Rescher and Ruth Manor in [23]. For this reason, I shall refer to it as "the Rescher-Manor Mechanism". Some interesting extensions, especially with respect to preferential mechanisms, are found in [21]. All the results may be easily rephrased in the semantic terms of [22], even if this is not done in the book itself. ${ }^{1}$ Still later, further consequence relations were added. An overview and comparison is presented in [14].

The inspiration that lead to inconsistency-adaptive logics is significantly different. The original paradigmatic application was the case where a theory was intended as consistent but turned out to be inconsistent. The idea was to find a logic that interprets the inconsistent theory 'as consistently as possible'. The consequence set defined by the logic should enable one to understand the premises by deriving consequences from them, to localize the inconsistencies derivable from them, and thus to prepare for the elimination of the inconsistencies (in view of extralogical considerations). Classical Logic - henceforth CL - assigns the trivial consequence set to inconsistent sets, whereas paraconsistent logics assign them a consequence set that is arguably too weak - see [5, p. 190].

When, around 1980, I obtained the first inconsistency-adaptive logic, ${ }^{2}$ the comparison with the Rescher-Manor Mechanism imposed itself - see Section 10 of [5]. First, that Mechanism does not seem to be a "logic": a formal system that may be presented in terms of a set of rules (and possibly axioms $^{3}$ ) that are closed under Uniform Substitution. Next, inconsistencyadaptive logics approach sets of premises in terms of dynamic proofs and in terms of sets of (possibly inconsistent) models, whereas the Rescher-Manor

\footnotetext{
${ }^{1}$ The relevant remark in [14, p. 20] is (to say the least) confusing. For example, $A$ is a Strong Consequences of $\Gamma$ iff it is verified by the schematization of the superpositions that verify $\Gamma$. Similarly for the other consequence relations.

${ }^{2}$ Unfortunately, [5] was only published in 1989; but later papers on the topic appeared earlier, e.g., [2], [3] and [4].

${ }^{3}$ Where a rule like Addition $(A / A \vee B)$ corresponds to the permission to add $A \vee B$ to a proof on the condition that it contains $A$, an axiom schema like $(A \& B) \supset A$ corresponds to the permission to add a formula of the form $(A \& B) \supset A$ to a proof on no condition at all.
} 
Mechanism approaches them in terms of the $\mathbf{C L}$-consequences of their $M C S$. The latter requires, first, that we are able to locate the $M C S$ - an often precarious enterprise at the predicative level - and next, that we combine their CL-consequence sets in some or other (more or less complex) way. Finally, the consequence sets of both approaches turn out to be in general drastically different.

Meanwhile, a multiplicity of adaptive logics have been devised and studied. Some, such as Priest's $\mathbf{L P}^{m}$ from [19], depart from a rather different philosophical viewpoint. Some, like Meheus' ANA from [17], unconditionally retain all analyzing rules from $\mathbf{C L}$, including the Disjunctive Syllogism. Many are not even paraconsistent, but tolerate other logical abnormalities. ${ }^{4}$ For an overview I refer to [10]. All this, however, does not clarify the relation between the Rescher-Manor Mechanism and adaptive logics. Adaptive logics form a nicely related field, with variations along several dimensions, but the Rescher-Manor Mechanism does not seem to fit in that field.

In this paper, I shall show that each consequence relation that was defined within the Rescher-Manor Mechanism is a particular inconsistencyadaptive logic. As we shall see in Section 4, gaining this insight was largely a matter of serendipity: ${ }^{5}$ A result of Guido Vanackere, who was working on a very different problem, offered the clue to the unification.

The gain, however, is more than just the unification. Adaptive logics have a dynamic proof theory, a (static) semantics, and a semantics that captures the dynamics of the proofs. The same henceforth holds for the Rescher-Manor Mechanism. Especially the gain of a dynamic proof theory seems important to me. Henceforth one is able to construct proofs from the premises in agreement with the Rescher-Manor Mechanism, even at the predicative level. Even if the consequence set is not decidable, one may take provisional decisions that are rational in the sense that they rely on the way things are to the best of one's (present) insights. ${ }^{6}$

\footnotetext{
${ }^{4}$ In $\mathbf{C L}$, all logical abnormalities surface as inconsistencies. For example, the premises $p \& q$ and $\sim p$ have an inconsistent CL-consequence set, but triviality may be avoided by turning Simplification into a conditional rule (or by making the corresponding axiom conditionally valid).

${ }^{5}$ Serendipity is often misinterpreted: if I had not been worrying about the problem for almost twenty years, I would not have seen the solution.

${ }^{6}$ In [20, p. 74] a proof-theoretic idea is present (premises and non-discharged hypotheses have to be mutually consistent). Carrying out such proofs requires that the consistency of such sets be decidable. Precisely this restriction is overcome by the result of the present paper.
} 
In the sequel of this paper, I briefly summarize the Rescher-Manor Mechanism (Section 2), introduce some adaptive logics that I shall need to make my point (Section 3), prove that the consequence relations defined within the Rescher-Manor Mechanism are adaptive logics (Section 4), outline the dynamic proofs for those consequence relations (Section 5), discuss the gain obtained by the unification, and comment on the applicability of the involved inconsistency-adaptive logics (Section 6).

A final warning: space limitations require that I deal only with the socalled flat case. Hence, I shall not discuss the way in which non-logical preferences may be integrated in the present framework. This is a small limitation: any competent reader may easily work out the matter for himself or herself.

\section{The Rescher-Manor mechanism}

Any set of sentences $\Gamma$ has one or more $M C S$. If the consistent members of $\Gamma$ are mutually consistent, there is one (the empty set iff $\Gamma$ has no consistent members). Otherwise there are more. Let $M C S(\Gamma)$ denote the set of $M C S$ of $\Gamma$.

Definition 2.1. $\Gamma \vdash_{\text {Strong }} A$ ( $A$ is a Strong Consequence of $\Gamma$ ) iff $\Delta \vdash_{\mathbf{C L}} A$ for all $\Delta \in M C S(\Gamma)$.

Definition 2.2. $\Gamma \vdash_{\text {Weak }} A$ ( $A$ is a Weak Consequence of $\Gamma$ ) iff $\Delta \vdash_{\mathbf{C L}} A$ for some $\Delta \in M C S(\Gamma)$.

Strong Consequences are sometimes called Universal Consequences, $M C$ consequences, or Inevitable Consequences. Weak Consequences are sometimes called Existential Consequences. For all $\Gamma,{ }^{7} C n_{\text {Strong }}(\Gamma)$ is consistent. $C n_{\text {Weak }}(\Gamma)$ is consistent iff $\left\{A \mid A \in \Gamma ; \nvdash_{\mathbf{C L}} \sim A\right\}$ is consistent.

DEFINITION 2.3. $\Delta \subseteq \Gamma$ is minimally inconsistent iff $\Delta$ is inconsistent and all $\Theta \subset \Delta$ are consistent.

Definition 2.4. $\operatorname{Inc}(\Gamma)=\{A \mid A \in \Delta$ for some minimally inconsistent $\Delta \subseteq \Gamma\}$.

Definition 2.5. $A \in \operatorname{Free}(\Gamma)$ iff $A \in \Gamma-\operatorname{Inc}(\Gamma)$.

It is easily seen that $A \in \operatorname{Free}(\Gamma)$ iff $A \in \Delta$ for any $\Delta \in M C S(\Gamma)$, and also that $A \in \operatorname{Free}(\Gamma)$ iff (i) $A \in \Gamma$ and (ii) for all $\Delta \subseteq \Gamma, \Delta \cup\{A\}$ is inconsistent iff $\Delta$ is.

\footnotetext{
${ }^{7} C n_{\text {Strong }}(\Gamma)=\left\{A \mid \Gamma \vdash_{\text {Strong }} A\right\}$ and similarly for other consequence relations.
} 
Definition 2.6. $\Gamma \vdash_{\text {Free }} A$ ( $A$ is a Free Consequence of $\Gamma$ ) iff Free $(\Gamma) \vdash_{\mathbf{C L}}$ A.

The search for a consequence set that is in general richer than $C n_{\text {Strong }}(\Gamma)$, but, unlike $C n_{W e a k}(\Gamma)$, does not contain flat inconsistencies (such as $A$ and $\sim A$ ), led to the following definitions.

Definition 2.7. $\Delta \subseteq \Gamma$ is an argument for $A$ iff (i) $\Delta$ is consistent, (ii) $\Delta \vdash_{\mathbf{C L}} A$, and (iii) for all $B \in \Delta, \Delta-\{B\} \nvdash_{\mathbf{C L}} A$.

Definition 2.8. $\Gamma \vdash_{\text {Argued }} A$ ( $A$ is an Argued Consequence of $\Gamma$ ) iff some $\Delta \subseteq \Gamma$ is an argument for $A$, whereas no $\Delta \subseteq \Gamma$ is an argument for $\sim A$.

A simpler and more perspicuous definition derives from Lemma 4.19 below: $A$ is an Argued Consequence of $\Gamma$ iff $A$ is a Weak Consequence of $\Gamma$ whereas its negation is not.

The last consequence relation to be considered is based on a 'numerical' criterion, whence I shall call it the C-Based (cardinality based) Consequence relation. Where $\Delta^{\#}$ is the cardinality of $\Delta$, let $C M C S(\Gamma)$ denote the set of $\Delta \in M C S(\Gamma)$ such that, for all $\Theta \in M C S(\Gamma), \Delta^{\#} \geq \Theta^{\#}$.

Definition 2.9. $\Gamma \vdash_{C \text {-Based }} A\left(A\right.$ is a $C$-Based Consequence of $\Gamma$ ) iff $\Delta \vdash_{\mathrm{CL}}$ $A$ for all $\Delta \in C M C S(\Gamma)$.

Most sensible applications of the C-Based Consequence relation require that $\Gamma$ is considered as a multiset (for which it makes a difference how many times a wff occurs in it).

It is easily seen (and proved in [14]) that all Free consequences of $\Gamma$ are Strong Consequences of $\Gamma$, that all of the latter are Argued Consequences of $\Gamma$ as well as C-Based Consequences of $\Gamma$, that all Argued Consequences of $\Gamma$ and all C-Based Consequences of $\Gamma$ are Weak Consequences of $\Gamma$, that all of the latter are $\mathbf{C L}$-consequences of $\Gamma$, whereas the converse of neither of these holds generally.

\section{Inconsistency-adaptive logics based on CLuN}

Two inconsistency-adaptive logics will be introduced here. Both have CL as their 'upper limit' logic (the logic that defines logical normality), and CLuN as their 'lower limit' logic (this defines the consequences that obtain unconditionally). They differ from each other in the strategy to interpret the phrase 'as consistently as possible'. This strategy determines the conditions 
under which an inference that holds according to CL but does not hold according to $\mathbf{C L u N}$, may be applied to specific members and consequences of the premises.

CLuN is a poor and basic paraconsistent logic (the first one I devised at the propositional level - see [1], where the logic is called PI). ACLuN1 and ACLuN2 are the oldest adaptive logics. The propositional proof theory of the former was first presented (by the name DDL) in [5]; the propositional semantics of the second was first presented in [3]. The predicative versions were studied in [8]. In view of these publications, I briefly summarize the matter here.

\subsection{The paraconsistent logic CLuN}

In $\mathbf{C L}$, the semantic clause for negation comes to the consistency requirement (if $v_{M}(A)=1$, then $v_{M}(\sim A)=0$ ) and the (negation-)completeness requirement (if $v_{M}(A)=0$, then $v_{M}(\sim A)=1$ ). As a proof theoretic counterparts of these, one may choose, for example, $A \supset(\sim A \supset B)$ and $A \vee \sim A$ respectively. The idea behind the paraconsistent logic CLuN is that we drop the consistency requirement. So, CLuN is the full positive part of CL, extended with $A \vee \sim A$ (or $(A \supset \sim A) \supset \sim A$ ). It is worth pointing out that the Replacement of Equivalents and the Replacement of Identicals have to be restricted to cases where the replacement takes place outside the scope of a " ". Also, many negation properties from CL are lost: both directions of Double Negation, de Morgan properties, etc. CLuN maximally isolates inconsistencies.

Introducing bottom (characterized by the axiom $\perp \supset A$ ) is useful. It not only simplifies metatheoretic proofs, but also enables us to define classical negation (by $\neg A==_{\text {df }} A \supset \perp$ ), which turns $\mathbf{C L u N ~ i n t o ~ C L ~ e x t e n d e d ~ w i t h ~}$ the very poor paraconsistent negation " $\sim$ ".

Here is a nice semantics for CLuN. It handles the quantifiers in a simple way and is very general (including uncountable models, etc.) We introduce a pseudo-language schema $\mathcal{L}^{+}$that extends the language $\mathcal{L}$ of $\mathbf{C L}$ with a (possibly uncountable) set $\mathcal{O}$ of pseudo-constants, and require that each element of the domain $D$ is named by either a constant or a pseudo-constant. Let $\mathcal{S}, \mathcal{C}, \mathcal{P}^{r}$, and $\mathcal{W}$ be, respectively, the set of sentential letters, constants, predicates of rank $r$, and wffs of $\mathcal{L}$. Let $\mathcal{W}^{+}$the set of wffs of $\mathcal{L}^{+}$, and let $\sim \mathcal{W}^{+}=\left\{\sim A \mid A \in \mathcal{W}^{+}\right\}$.

A model $M=\langle D, v\rangle$ is an interpretation of $\mathcal{W}^{+}$, and hence of $\mathcal{W}$, which is what we are interested in. The assignment function $v$ is defined by: 
$\mathrm{C} 1.1 \quad v: \mathcal{S} \longrightarrow\{0,1\}$

$\mathrm{C} 1.2 v: \mathcal{C} \cup \mathcal{O} \longrightarrow D($ where $D=\{v(\alpha) \mid \alpha \in \mathcal{C} \cup \mathcal{O}\})$

C1.3 $v: \mathcal{P}^{r} \longrightarrow \wp\left(D^{r}\right)$ (the power set of the $r$-th Cartesian product of $D$ )

C1.4 $v: \sim \mathcal{W}^{+} \longrightarrow\{0,1\}$

The valuation function $v_{M}$ determined by $M$ is defined as follows:

$\mathrm{C} 2.1 v_{M}: \mathcal{W}^{+} \longrightarrow\{0,1\}$

C2.2 where $A \in \mathcal{S}, v_{M}(A)=v(A) ; v_{M}(\perp)=0$

$\mathrm{C} 2.3 v_{M}\left(\pi^{r} \alpha_{1} \ldots \alpha_{r}\right)=1$ iff $\left\langle v\left(\alpha_{1}\right), \ldots, v\left(\alpha_{r}\right)\right\rangle \in v\left(\pi^{r}\right)$

$\mathrm{C} 2.4 v_{M}(\alpha=\beta)=1$ iff $v(\alpha)=v(\beta)$

$\mathrm{C} 2.5 v_{M}(\sim A)=1$ iff $v_{M}(A)=0$ or $v(\sim A)=1$

$\mathrm{C} 2.6 v_{M}(A \supset B)=1$ iff $v_{M}(A)=0$ or $v_{M}(B)=1$

$\mathrm{C} 2.7 \quad v_{M}(A \& B)=1$ iff $v_{M}(A)=1$ and $v_{M}(B)=1$

$\mathrm{C} 2.8 v_{M}(A \vee B)=1$ iff $v_{M}(A)=1$ or $v_{M}(B)=1$

$\mathrm{C} 2.9 v_{M}(A \equiv B)=1$ iff $v_{M}(A)=v_{M}(B)$

$\mathrm{C} 2.10 v_{M}((\forall \alpha) A(\alpha))=1$ iff $v_{M}(A(\beta))=1$ for all $\beta \in \mathcal{C} \cup \mathcal{O}$

$\mathrm{C} 2.11 v_{M}((\exists \alpha) A(\alpha))=1$ iff $v_{M}(A(\beta))=1$ for some $\beta \in \mathcal{C} \cup \mathcal{O}$

Truth in a model, semantic consequence and validity are defined as usual. CLuN is provably Sound and Complete with respect to this semantics.

The following Lemmas and Theorem will prove useful in Section 4.

Lemma 3.1. $\Gamma$ is $\neg$-consistent iff it has a CLuN-model.

Proof. Obvious in view of the fact that, for any CLuN-model $M, v_{M}(\neg A)$ $=1$ iff $v_{M}(A)=0$.

Let $\mathcal{L}^{\nsim}$ be the $\sim$-free fragment of $\mathcal{L}$ and let $\mathcal{W}^{\nsim}$ be the set of wffs of $\mathcal{L}^{\nsim}$. Lemma 3.2. If a CL-model $M$ and a $\mathbf{C L u N - m o d e l ~} M^{\prime}$ agree with respect to $C 1.1-3$ of the semantics, then $v_{M}(A)=v_{M^{\prime}}(A)$ for all $A \in \mathcal{W}^{\nsim}$.

Proof. If $A \in \mathcal{W}^{\nsim}, \mathrm{C} 1.4$ does not interfere with $v_{M^{\prime}}(A)$.

Hence, the proof of the following Theorem is obvious:

Theorem 3.1. If $(\Gamma \cup\{A\}) \subseteq \mathcal{W}^{\nsim}$, then $\Gamma \vDash_{\mathbf{C L}} A$ iff $\Gamma \vDash_{\mathbf{C L u N}} A$.

Lemma 3.3. If $\Gamma \subseteq \mathcal{W}^{\nsim}$ and $\Delta$ is a set of wffs of the form $\sim A$, then $\Gamma$ is $\neg$-consistent iff $\Gamma \cup \Delta$ is $\neg$-consistent. 
Proof. If $\Gamma \cup \Delta$ is $\neg$-consistent, so is $\Gamma$. So suppose that $\Gamma \subseteq \mathcal{W}^{\nsim}$ is $\neg$ consistent. By Lemma 3.1, $\Gamma$ has a CLuN-model $M=\langle D, v\rangle$. As $\Gamma \subseteq \mathcal{W}^{\nsim}$, we may suppose that $v(\sim A)=0$ for all $A .^{8}$ Let $M^{\prime}=\left\langle D, v^{\prime}\right\rangle$ be exactly as $M$ except in that $v(\sim A)=1$ iff $A \in \Delta . M^{\prime}$ verifies $\Gamma \cup \Delta$ and hence this set is $\neg$-consistent.

\subsection{Abstract characterization of the adaptive logics}

Although the discovery of adaptive logics proceeded in terms of dynamic proofs, and although the main results of this paper are an outcome of insights in such proofs, it will be easier for the reader that I start with an abstract characterization of the adaptive logics. This characterization is in terms of CLuN-models and in terms of CLuN-derivability.

All statements made below are proved in [5] for the propositional level and, more importantly, in [8] for the full predicative logics. Let ! $A$ abbreviate $A \& \sim A$, let $\exists A$ abbreviate $A$ preceded (in some definite order) by an existential quantifier over any variable free in $A$, and let $D E K\left\{A_{1}, \ldots, A_{n}\right\}$ abbreviate $\exists ! A_{1} \vee \cdots \vee \exists ! A_{n}$. It was shown in [8] that:

Theorem 3.2. $\vdash_{\mathbf{C L}}\left(A_{1} \& \cdots \& A_{n}\right) \supset B$, iff there are $C_{1}, \ldots, C_{m}$ such that $\vdash_{\mathrm{CLuN}} D E K\left\{C_{1}, \ldots, C_{m}\right\} \vee\left(\left(A_{1} \& \cdots \& A_{n}\right) \supset B\right)$.

Henceforth, it will be easier to write $\operatorname{DEK}(\Delta)$, recalling that this is a formula and hence that $\Delta$ is finite.

I now first define the reliability strategy. Applied to $\mathbf{C L u N}$, it leads to the inconsistency-adaptive logic ACLuN1.

Definition 3.10. DEK $(\Delta)$ is a minimal DEK-consequence of $\Gamma$ iff $\Gamma \vDash_{\mathbf{C L u N}} \operatorname{DEK}(\Delta)$ and, for no $\Theta \subset \Delta, \Gamma \vDash_{\mathbf{C L u N}} \operatorname{DEK}(\Theta)$.

DEFINITION 3.11. The set of formulas that are unreliable with respect to $\Gamma$ : $U(\Gamma)=\{A \mid A \in \Delta$ for some minimal $D E K$-consequence $D E K(\Delta)$ of $\Gamma\}$.

Definition 3.12. The set of abnormalities in $M: A b(M)={ }_{\mathrm{df}}\{A \mid$ $\left.v_{M}(\exists ! A)=1\right\}$

Definition 3.13. $M$ is an ACLuN1-model of $\Gamma$ iff $A b(M) \subseteq U(\Gamma)$.

Definition 3.14. $\Gamma \vDash_{\text {ACLuN1 }} A$ ( $A$ is a final ACLuN1-consequence of $\Gamma$ ) iff all ACLuN1-models of $\Gamma$ verify $A$.

\footnotetext{
${ }^{8}$ If this is not the case, just transform the model to make it the case; this changes nothing to $v_{M}(A)$ for any $A \in \Gamma$.
} 
Given the Soundness and Completeness of CLuN, the syntactic counterpart of these definitions is immediate:

Definition 3.15. $\Gamma \vdash_{\text {ACLuN1 }} A$ ( $A$ is finally ACLuN1-derivable from $\Gamma$ ) iff there are $C_{1}, \ldots, C_{n}$ such that $\Gamma \vdash_{\mathbf{C L u N}} \operatorname{DEK}\left\{C_{1}, \ldots, C_{n}\right\} \vee A$ and $\left\{C_{1}, \ldots, C_{n}\right\} \cap U(\Gamma)=\emptyset$.

The minimal abnormality strategy selects a smaller set of CLuN-models:

Definition 3.16. $M$ is an ACLuN2-model (a minimally abnormal model) of $\Gamma$ iff there is no CLuN-model $M^{\prime}$ of $\Gamma$ such that $A b\left(M^{\prime}\right) \subset A b(M)$.

Definition 3.17. $\Gamma \vDash_{\text {ACLuN2 }} A$ ( $A$ is a final ACLuN2-consequence of $\Gamma$ ) iff all ACLuN2-models of $\Gamma$ verify $A$.

Again, the syntactic counterpart is immediate, but somewhat complex. What we are interested in is an economic syntactic characterization of the inconsistencies that are verified by the minimally abnormal models. Let $A<$ $B$ indicate that $\exists B$ is obtained from $\exists A$ either by relettering the individual variables or by Existential Generalization. Let $\Phi_{\Gamma}$ be such that, for any $\phi \in \Phi_{\Gamma}$, (i) the variables in each $A \in \phi$ occur in alphabetical order $(x, y, z$, $x_{1}, y_{1}, \ldots$ ), (ii) if $D E K(\Delta)$ is a minimal $D E K$-consequence of $\Gamma$, then for each $B \in \Delta$ there is a $A \in \phi$ such that $A<B$, and (iii) if $\phi, \phi^{\prime} \in \Phi_{\Gamma}$ and, for each $A \in \phi$ there is a $B \in \phi^{\prime}$ such that $A<B$, then $\phi=\phi^{\prime} .{ }^{9}$

Definition 3.18. $\Gamma \vdash_{\text {ACLuN2 }} A$ ( $A$ is a finally ACLuN2-derivable from $\Gamma)$ iff, for all $\phi \in \Phi_{\Gamma}$, there is a finite $\Delta$ such that $\phi \cap \Delta=\emptyset$ and $\Gamma \vdash_{\text {CLuN }}$ $A \vee D E K(\Delta)$.

Needless to say, an adaptive logic is sensible only if any $\Gamma$ that has paraconsistent $(\mathbf{C L u N}-)$ models also has adaptive models (Reassurance). If Reassurance would fail, the adaptive consequence set of $\Gamma$ might be trivial (in that $\Gamma$ has no adaptive models), whereas the $\mathbf{C L u N}$-consequence set of $\Gamma$ is not trivial. We can not only prove Reassurance, but something much stronger, viz.

Theorem 3.3. For any CLuN-model $M$ of $\Gamma$ there is an ACLuN2-model $M^{\prime}$ of $\Gamma$ such that $A b\left(M^{\prime}\right) \subseteq A b(M)$. (Strict Reassurance)

\footnotetext{
${ }^{9}$ This rules out $\phi \subset \phi^{\prime}$. See [8] for a more constructive definition. $\Phi_{\Gamma}=\emptyset$ iff $\Gamma$ is consistent.
} 
In other words: if $M$ is eliminated, then some (set-theoretically speaking) less abnormal model $M^{\prime}$ justifies its elimination. I refer to $[9]^{10}$ for the proof, and for the proof that Strict Reassurance also holds in ACLuN1 and in several other inconsistency-adaptive logics.

The thus defined ACLuN1 and ACLuN2 are provably Sound and Complete with respect to their semantics. Some further strategies have been devised - see, e.g., [15] — and there are many other paraconsistent (and other) logics to which these strategies may be applied. However, all this is not directly relevant for the present paper. Some new strategies will be presented in Section 4.

\subsection{Dynamic proofs}

The idea behind dynamic proofs is that a formula that is derivable at some stage of the proof may be underivable at a later stage, or vice versa. Adding a line to the proof at a stage may be justified with respect to our understanding of the premises at that stage; our understanding of the premises at a later stage may force us to 'delete' the line (actually, we shall mark it, indicating that it is OUT), and our understanding of the premises at a still later stage may force us to restore (unmark) the line. Our goal, of course, is to find out which formulas are finally derivable from the premises according to the chosen adaptive logic. So, we need to define the conditions under which some formula is finally derived in a proof.

Technically, we realize the dynamics by distinguishing between unconditionally and conditionally derived formulas. A line in a proof will contain (i) a line number, (ii) the wff derived, (iii) the numbers of the lines from which the wff is derived, (iv) the rule by which the wff is derived, and moreover ( $\mathrm{v}$ ) the set of wffs that need to 'behave consistently' on the premises in order for the wff to be derivable. This fifth element is the empty set iff the second element is derived unconditionally.

Marking and unmarking lines will proceed in terms of, respectively, reliability and minimal abnormality. Only, here we do not refer to the abstract definitions, but to the estimations of reliability or minimal abnormality in view of the stage of the proof.

Let me start with reliability. $A \in U_{s}(\Gamma), A$ is unreliable at stage $s$ of a proof from $\Gamma$, iff there is a $\Delta$ such that $A \in \Delta, D E K(\Delta)$ is derived unconditionally at stage $s$, and $D E K(\Theta)$ is not derived unconditionally at stage $s$ for

\footnotetext{
10 There it is also proved that the absence of Strict Reassurance has unpalatable consequences.
} 
any $\Theta \subset \Delta$. At stage $s$, we mark all lines the fifth element of which contains some member of $U_{s}(\Gamma)$. (On our insights at stage $s$ of the proof, $U_{s}(\Gamma)$ is the set of formulas that are unreliable with respect to $\Gamma$.) Here is an example of an ACLuN1-proof in terms of natural deduction rules (I skip their names, listing the fifth element of the line in the fourth column). I choose the example in view of the comparison with the consequence relations from the Rescher-Manor Mechanism.

$\begin{array}{lllll}1 & q & \text { PREM } & \emptyset & \\ 2 & \sim p \&(q \supset r) & \text { PREM } & \emptyset & \\ 3 & \sim r \vee s & \text { PREM } & \emptyset & \\ 4 & p \vee t & \text { PREM } & \emptyset & \\ 5 & p \vee u & \text { PREM } & \emptyset & \\ 6 & \sim q \vee t & \text { PREM } & \emptyset & \\ 7 & p \vee \sim q & \text { PREM } & \emptyset & \\ 8 & \sim p & 2 & \emptyset & \\ 9 & q \supset r & 2 & \emptyset & \\ 10 & r & 1,9 & \emptyset & \\ 11 & s & 3,10 & \{r\} & \\ 12 & t & 4,8 & \{p\} & \text { OUT } \\ 13 & u & 5,8 & \{p\} & \text { OUT } \\ 14 & t & 1,6 & \{q\} & \text { OUT } \\ 15 & ! p \vee ! q & 1,7,8 & \emptyset & \end{array}$

At stage 15, lines 12-14 are marked OUT because both $p$ and $q$ are unreliable at this stage. It is easily seen that they are unreliable at all later stages, and that no other wffs are unreliable on these premises. So, all wffs that occur on unmarked lines are finally ACLuN1-derivable from the premises, whereas those that occur on the marked lines are not. Remark that $s$ is ACLuN1-derivable but not $\mathbf{C L u N}$-derivable from the premises.

For the minimal abnormality strategy, we start again from the minimal $D E K$-formulas at stage $s$ (and reletter their disjuncts in such a way that the variables occur in alphabetic order). From these $D E K$-formulas we define $\Phi_{s}$ in the same way that $\Phi_{\Gamma}$ is defined from the minimal $D E K$-consequences of $\Gamma$ in subsection 3.2. Let $A$ be derived at some line. The line is marked at stage $s$ iff, for some $\phi \in \Phi_{s}$, there is no line at which $A$ is derived and the fifth element of which does not contain any element of $\phi$.

Applying this to the previous proof, $\Phi_{15}=\{\{p\},\{q\}\}$. Line 13 is marked at stage 15: $u$ is not derived at a line the fifth element of which does not contain $p$. Lines 12 and 14 (and all other lines) are unmarked. Again, it 
is easily seen that all formulas (including $t$ ) that occur on unmarked lines are finally ACLuN2-derivable from the premises, whereas $u$ is not. Actually, the premises illustrate the typical difference between ACLuN1 and ACLuN2. Think about it in semantic terms. $t$ is false in some ACLuN1models of the premises that verify both $! p$ and $! q$. However, $t$ is true in all ACLuN2-models of the premises. All of these verify either $! p$ or $! q$, but none of them verifies both.

Any $\mathbf{C L u N}$-consequence of $\Gamma$ can be unconditionally derived in an adaptive proof from $\Gamma$. If $A$ is a $\mathbf{C L}$-consequence of $\Gamma$ that is not a $\mathbf{C L u N -}$ consequence of $\Gamma, A$ can be conditionally derived in an adaptive proof from $\Gamma$. Whether, in the latter case, $A$ is or is not marked depends on the $D E K$ formulas derived at the stage of the proof, viz. of the stage's estimate of what is, respectively, reliable or minimally abnormal.

How do dynamic proofs relate to final derivability? For ACLuN1, $A$ is finally derived at line $i$ of the proof iff any (finite) extension of the proof can be further extended in such a way that line $i$ is unmarked. ${ }^{11}$ For ACLuN2, we have to require that even infinite extensions of the proof can be further extended in such a way that line $i$ is unmarked - see [8, p. 466] for details. All the fuss is avoided if the set of minimal $D E K$-consequences of $\Gamma$ is decidable. In general, however, criteria for deciding from a finite proof that $A$ is finally derived are important. Such criteria are offered in [11], [12], and [6].

As the semantics only defines the final consequence relation, some people questioned whether the dynamics of the proofs is real. This has been settled in [7] and especially in [6] (that also contains other new insights from dynamic semantics). Any stage of a dynamic proof is adequate with respect to a block semantics in which the 'blocks' are defined by the stage of the proof. This justifies that we consider the dynamic proofs as sensible formal mechanisms (that are close to our actual reasoning).

\section{The unification}

The example from the previous Section illustrates nicely that the consequence sets delivered by CLuN, ACLuN1, and ACLuN2 are quite distinct from those delivered by the consequence relations defined within the Rescher-Manor Mechanism. It is also instructive to see how the adaptive

11 If the fifth element of line $i$ is $\Delta, D E K(\Theta)$ is unconditionally derivable from the premises, but has not been derived in the proof, one may derive $D E K(\Theta \cup\{B\})$ for some $B \in \Delta$, thus forcing line $i$ to be marked. By later deriving $D E K(\Theta)$, line $i$ is again unmarked. 
logics behave with respect to the properties studied in [14]. ${ }^{12}$ Limitations of space force me to leave all this to the reader.

Up to this point, I have always presupposed that logical constants from a natural language have to be formalized in terms of $\sim, \&, \vee, \supset, \equiv,=, \forall$, and $\exists$. Of course, some further logical constants may be defined in CLuN. We already met $\neg$. A further interesting case is $A \sqsupset B=_{\mathrm{df}} \sim A \vee B$. This is a candidate for formalizing implications - see [27] for a mixed use of $\supset$ and $\sqsupset$ in the adaptive interpretation of an inconsistent arithmetic. If the premises are inconsistent, $\neg$ does not seem to have any use at all. Still, where adaptive logics are varied by varying the 'lower limit' paraconsistent logic and the adaptive strategy, the application of an adaptive logic to natural language premises may obviously be varied by varying the interpretation of the logical constants that occur in the premises. ${ }^{13}$

In an attempt to generalize the approach to preferences from his [25], Guido Vanackere arrived at a completely different interpretation of the premises - see [26]. The procedure (called 'Guido's trick' in Ghent) is to formalize the negations in the premises by $\neg$, and to precede each premise by $\sim \neg$. The trick is rather ingenious. Suppose that the sentence in natural language reads " $p$ or not $q$ ". This becomes:

$$
\sim \neg(p \vee \neg q)
$$

which is $\mathbf{C L u N - e q u i v a l e n t ~ t o ~}$

$$
\sim \neg(p \vee \neg q) \&(\neg(p \vee \neg q) \vee(p \vee \neg q))
$$

and hence also to

$$
! \neg(p \vee \neg q) \vee(p \vee \neg q)
$$

In an adaptive logic obtained from $\mathbf{C L u N}$,

$$
p \vee \neg q
$$

is derivable from the previous on the condition that $\neg(p \vee \neg q)$ behaves [ ]consistently. It was this insight that triggered the present results.

\footnotetext{
12 Some of those properties are defined in a way that presupposes the Rescher-Manor Mechanism. If those restrictions are removed, it is easily seen that the adaptive consequence relations are insensitive with respect to duplication, local consequence, universal consequence and clausal form (if the latter three are defined in terms of CLuN).

${ }^{13}$ In a broader sense of the term, a logic consists of a formal system and a link between the latter and natural languages. In that sense, varying the link leads to a different logic.
} 
I shall show that each consequence relation from the Rescher-Manor Mechanism is characterized by an adaptive logic that has CLuN as its lower limit logic. To keep the exposition simple, I suppose from now on that the Rescher-Manor Mechanism is formulated in terms of $\mathcal{L}^{\nsim}$ - please take this into account when consulting Section 2.

If we extend $\mathcal{L}^{\nsim}$ (the language of $\mathbf{C L}$ in the present context) to $\mathcal{L}$ (the language of $\mathbf{C L u N}$ ), we can define, the Guido-transformation of $\Gamma$, viz. $\Gamma^{G}=\{\sim \neg A \mid A \in \Gamma\}$. For any of the consequence relations $\Gamma \vdash_{X} A$ defined within the Rescher-Manor Mechanism, I shall show that there is an adaptive logic ACLuN $i$ such that $\Gamma \vdash_{X} A$ iff $\Gamma^{G} \vdash_{\text {ACLuN } i} A$. As far as dynamic proofs are concerned, the correspondence may be made even more striking (see footnote 16).

I now come to the Lemmas and Theorems. I suppose throughout that $\Gamma$ is a set of wffs of $\mathcal{L}^{\nsim}$.

\subsection{Free consequences - reliability}

Lemma 4.4. For all $\Delta \subseteq \Gamma, \Delta$ is $\neg$-consistent iff some $\mathbf{C L u N}$-model of $\Gamma^{G}$ verifies $\Delta$.

Proof. Let $\Delta \subseteq \Gamma \subseteq \mathcal{L}^{\nsim}$. It is easily seen that each of the following are equivalent: $\Delta$ is $\neg$-consistent; $\Gamma^{G} \cup \Delta$ is $\neg$-consistent (by Lemma 3.3); $\Gamma^{G} \cup \Delta$ has a $\mathbf{C L u N - m o d e l ; ~ s o m e ~} \mathbf{C L u N}$-model of $\Gamma^{G}$ verifies $\Delta$.

Lemma 4.5. If $A \in \Gamma$, then any $\mathbf{C L u N - m o d e l ~ o f ~} \Gamma^{G}$ verifies $A$ iff it falsifies $! \neg A$.

Proof. Let $M$ be a CLuN-model of $\Gamma^{G}$ and $A \in \Gamma$. It follows that $M$ verifies $\sim \neg A$. So, $M$ verifies $! \neg A$ iff it verifies $\neg A$; that is, iff it falsifies $A$.

Lemma 4.6. $\Delta \subseteq \Gamma$ is minimally $\neg$-inconsistent iff $D E K\{\neg A \mid A \in \Delta\}$ is a minimal $D E K$-consequence of $\Gamma^{G}$.

Proof. Each of the following are equivalent:

$-\Delta \subseteq \Gamma$ is minimally $\neg$-inconsistent.

- $\Delta$ is $\neg$-inconsistent and no subset of $\Delta$ is $\neg$-inconsistent (by Definition 2.3).

- No CLuN-model of $\Gamma^{G}$ verifies $\Delta$, and, for each $B \in \Delta$, some CLuNmodel of $\Gamma^{G}$ verifies $\Delta-\{B\}$ (by Lemma 4.4).

- Each CLuN-model of $\Gamma^{G}$ verifies $! \neg B$ for some $B \in \Delta$, and, for each $B \in \Delta$, some CLuN-model of $\Gamma^{G}$ falsifies $D E K\{\neg A \mid A \in \Delta-\{B\}\}$ (by Lemma 4.5). 
- $D E K\{\neg A \mid A \in \Delta\}$ is a minimal $D E K$-consequence of $\Gamma^{G}$ (by Definition 3.10).

Lemma 4.7. $A \in \operatorname{Free}(\Gamma)$ iff $A \in \Gamma$ and $\neg A \notin U\left(\Gamma^{G}\right)$.

Proof. By Definitions 2.4 and 2.5, $A \in$ Free $(\Gamma)$ iff $A \in \Gamma-\Delta$ for all minimally $\neg$-inconsistent $\Delta \subseteq \Gamma$. In view of Lemma 4.6, the latter is equivalent to: $A \in \Gamma-\Delta$ for all minimal $D E K$-consequences $D E K\{\neg A \mid A \in \Delta\}$ of $\Gamma^{G}$. By Definition 3.11, this is equivalent to $A \in \Gamma$ and $\neg A \notin U\left(\Gamma^{G}\right)$.

Lemma 4.8. Any ACLuN1-model of $\Gamma^{G}$ verifies Free $(\Gamma)$.

Proof. Let $M$ be an ACLuN1-model of $\Gamma^{G}$. Hence, $A b(M) \subseteq U\left(\Gamma^{G}\right)$ (Definition 3.13). Consider any $A \in$ Free $(\Gamma)$. In view of Lemma 4.7, $A \in \Gamma$ and $\neg A \notin A b(M)$; hence $M$ falsifies $\neg \neg A$. But then $M$ verifies $A$ in view of Lemma 4.5.

Theorem 4.4. If $\Gamma \cup\{A\} \subseteq \mathcal{W}^{\nsim}, \Gamma \vdash_{\text {Free }} A$ iff $\Gamma^{G} \vDash_{\text {ACLuN1 }} A$.

Proof. Let $\Gamma \cup\{A\} \subseteq \mathcal{W}^{\nsim}$. Suppose first that $\Gamma \vdash_{\text {Free }} A$. Hence, $\operatorname{Free}(\Gamma) \vDash_{\mathbf{C L}} A$. Let $M$ be any ACLuN1-model of $\Gamma^{G}$. By Lemma 4.8, $M$ verifies Free $(\Gamma)$. Hence, by Theorem $3.1, M$ verifies $A$. So, $\Gamma^{G} \vDash_{\text {ACLuN1 }} A$.

Suppose next that $\Gamma \nvdash_{\text {Free }} A$. By Definition 2.6, some CL-model $M=$ $\langle D, v\rangle$ of $\operatorname{Free}(\Gamma)$ falsifies $A$. Let $M^{\prime}=\left\langle D, v^{\prime}\right\rangle$ be a CLuN-model obtained from $M$ by setting $v^{\prime}$ identical to $v$ for C1.1-3, and by setting $v^{\prime}(\sim B)=1$ iff $\sim B \in \Gamma^{G}$. For all $B \in \Gamma^{G}, v^{\prime}(B)=1$ and hence $v_{M^{\prime}}(B)=1$. So:

(i) $M^{\prime}$ verifies $\Gamma^{G}$.

From $A \in \mathcal{W}^{\nsim}$ and $M$ falsifies $A$ by Lemma 3.2:

(ii) $M^{\prime}$ falsifies $A$.

Similarly, as $M$ verifies $\operatorname{Free}(\Gamma)$ and $\operatorname{Free}(\Gamma) \subseteq \mathcal{W}^{\nsim}$,

(iii) $M^{\prime}$ verifies Free $(\Gamma)$.

But then, for all $C \in \operatorname{Free}(\Gamma), M^{\prime}$ falsifies $\neg C$, and hence also $! \neg C$. So,

(iv) If $B \in A b\left(M^{\prime}\right)$, then $B \notin\{\neg C \mid C \in \operatorname{Free}(\Gamma)\}$;

By the definition of $v^{\prime}$ and clause C2.5 of the CLuN-semantics:

(v) If $B \in A b\left(M^{\prime}\right)$, then $B \in\{\neg C \mid C \in \Gamma\}$.

Consider any $B \in A b\left(M^{\prime}\right)$. By (iv) and (v): for some $C, B=\neg C, C \in \Gamma$ and $C \notin \operatorname{Free}(\Gamma)$. But then $B \in U(\Gamma)$ (by Lemma 4.7). So, $A b\left(M^{\prime}\right) \subseteq U(\Gamma)$. Hence, by Definition 3.13,

(vi) $M^{\prime}$ is an ACLuN1-model of $\Gamma^{G}$.

Hence, in view of (i), (ii), and (vi), $\Gamma^{G} \not{ }_{\text {ACLuN1 }} A$. 


\subsection{Strong consequences - minimal abnormality}

Lemma 4.9. If $M$ and $M^{\prime}$ are CLuN-models of $\Gamma^{G}, A b\left(M^{\prime}\right) \subseteq A b(M)$, $A \in \Gamma$, and $M$ verifies $A$, then $M^{\prime}$ verifies $A$.

Proof. Suppose that the antecedent is true. As $M$ verifies $A$, it falsifies $! \neg A$ (by Lemma 4.5). As $A b\left(M^{\prime}\right) \subseteq A b(M), M^{\prime}$ falsifies $! \neg A$. But then, in view of Lemma $4.5, M^{\prime}$ verifies $A$.

Lemma 4.10. If $\Delta \in M C S(\Gamma)$, then there is a minimally abnormal CLuNmodel $M$ of $\Gamma^{G}$ such that $\Delta=\left\{A \mid A \in \Gamma ; v_{M}(A)=1\right\}$.

Proof. Suppose that $\Delta \in M C S(\Gamma)$. For all $A \in \Gamma-\Delta, \Delta \vDash_{\mathbf{C L}} \neg A$. Let $M^{\prime}$ be a CL-model of $\Delta$ (there is one as $\Delta$ is $\neg$-consistent). $\Delta=\{A \mid A \in$ $\left.\Gamma ; v_{M^{\prime}}(A)=1\right\}$, and $v_{M^{\prime}}(\neg A)=1$ for all $A \in \Gamma-\Delta$.

Let $M$ be a CLuN-model that agrees with $M^{\prime}$ in $\mathrm{C} 1.1-3$ and is such that $v(\sim A)=1$ iff $\sim A \in \Gamma^{G}$. By Lemma 3.2, $M$ verifies $\Delta$ and $M$ verifies $\neg A$ for all $A \in \Gamma-\Delta$. Moreover, $M$ verifies $\sim \neg A$ iff $\sim \neg A \in \Gamma^{G}$. It follows that $A b(M)=\{\neg A \mid A \in \Gamma-\Delta\}$ and that $\Delta=\left\{A \mid A \in \Gamma ; v_{M}(A)=1\right\}$.

Suppose that there were a $\mathbf{C L u N - m o d e l ~} M^{\prime \prime}$ of $\Gamma^{G}$ such that $A b\left(M^{\prime \prime}\right) \subset$ $A b(M)$. There then is an $A \in \Gamma-\Delta$ such that $M^{\prime \prime}$ falsifies $\neg \neg$. Being a CLuN-model of $\Gamma^{G}, M^{\prime \prime}$ verifies $\sim \neg A$, and hence falsifies $\neg A$ and verifies $A$. By Lemma $4.9, M^{\prime \prime}$ verifies $\Delta$. So, $M^{\prime \prime}$ verifies $\Delta \cup\{A\}$. But this is impossible, as $\Delta \in M C S(\Gamma)$. Hence $M$ is a minimally abnormal CLuNmodel of $\Gamma^{G}$.

Lemma 4.11. If $M$ is a minimally abnormal CLuN-model of $\Gamma^{G}$, then $A b(M) \subseteq\{\neg A \mid A \in \Gamma\}$.

Proof. Suppose first that $M=\langle D, v\rangle$ is a minimally abnormal CLuNmodel of $\Gamma^{G}$. It follows that $v_{M}(\sim \neg A)=1$ for all $A \in \Gamma$. Suppose next that there is a $B \in A b(M)-\{\neg A \mid A \in \Gamma\}$. As $M$ verifies $! B, v(\sim B)=1$. Let $M^{\prime}=\left\langle D, v^{\prime}\right\rangle$ be exactly like $M$ except in that $v^{\prime}(\sim A)=1$ iff $v(\sim A)=1$ and $\sim A \in \Gamma^{G}$. As $\sim B \notin \Gamma^{G}, v^{\prime}(\sim B)=0$ and hence $v_{M^{\prime}}(! B)=0$. As some CLmodel agrees with both $M$ and $M^{\prime}$ with respect to $\mathrm{C} 1.1-3, v_{M}(A)=v_{M^{\prime}}(A)$ for all $A \in \mathcal{W}^{\nsim}$ (by Lemma 3.2).

For any $A \in \Gamma\left(\subseteq \mathcal{W}^{\nsim}\right), v_{M}(\sim \neg A)=1$ and hence $v_{M}(\neg A)=0$ or $v(\sim \neg A)=1$. So, $v_{M^{\prime}}(\neg A)=0$ or $v^{\prime}(\sim \neg A)=1$, and hence $v_{M^{\prime}}(\sim \neg A)=1$. It follows that $M^{\prime}$ is a model of $\Gamma^{G}$ and that $A b\left(M^{\prime}\right) \subset A b(M)$ (as $M$ verifies $! B$ whereas $M^{\prime}$ does not). But this contradicts the first supposition. 


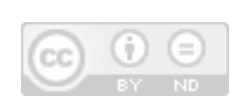

TOWARDS THE UNIFICATION ...

Lemma 4.12. If $M$ is a minimally abnormal CLuN-model of $\Gamma^{G}$, then $A b(M)=\left\{\neg A \mid A \in \Gamma ; v_{M}(A)=0\right\}$.

Proof. Suppose that $M$ is a minimally abnormal CLuN-model of $\Gamma^{G}$. By Lemma 4.11, $A b(M) \subseteq\{\neg A \mid A \in \Gamma\}$. If $v_{M}(A)=1$, then $\neg A \notin A b(M)$ (by Lemma 4.5). Hence $A b(M) \subseteq\left\{\neg A \mid A \in \Gamma ; v_{M}(A)=0\right\}$. But it follows from Lemma 4.5 that $\left\{\neg A \mid A \in \Gamma ; v_{M}(A)=0\right\} \subseteq A b(M)$.

Lemma 4.13. If $M$ is a minimally abnormal CLuN-model of $\Gamma^{G}$, then $\{A \mid$ $\left.A \in \Gamma ; v_{M}(A)=1\right\} \in M C S(\Gamma)$.

Proof. Suppose first that $M$ is a minimally abnormal CLuN-model of $\Gamma^{G}$. Let $\Delta=\left\{A \mid A \in \Gamma ; v_{M}(A)=1\right\}$. By Lemma $4.4, \Delta$ is $\neg$-consistent, and by Lemma $4.12, A b(M)=\{\neg A \mid A \in \Gamma-\Delta\}$.

Suppose next that $\Delta \notin M C S(\Gamma)$. Then there is a $\Theta$ such that $\Delta \subset$ $\Theta \in M C S(\Gamma)$. By Lemma 4.10, some minimally abnormal CLuN-model $M^{\prime}$ of $\Gamma^{G}$ is such that $\Theta=\left\{A \mid A \in \Gamma ; v_{M^{\prime}}(A)=1\right\}$. So, by Lemma 4.12, $A b\left(M^{\prime}\right)=\{\neg A \mid A \in \Gamma-\Theta\}$. As $\Delta \subset \Theta, A b\left(M^{\prime}\right) \subset A b(M)$. But this contradicts the first supposition.

Lemma 4.14. If $\Delta \in M C S(\Gamma)$, then $\Delta \vdash_{\mathrm{CL}} A$ iff $v_{M}(A)=1$ for all minimally abnormal CLuN-models $M$ of $\Gamma^{G}$ such that $\Delta=\left\{B \mid B \in \Gamma ; v_{M}(B)=1\right\}$.

Proof. Consider any $\Delta \in M C S(\Gamma)$. For the first direction, suppose that $\Delta \vdash_{\mathbf{C L}} A$, that $M$ is a minimally abnormal $\mathbf{C L u N}$-model of $\Gamma^{G}$, and that $\Delta=\left\{B \mid B \in \Gamma ; v_{M}(B)=1\right\}$. As $M$ is a CLuN-model of $\Delta$ and $\Delta \vdash_{\mathbf{C L}} A$, $M$ verifies $A$ by Theorem 3.1.

For the second direction, suppose that $M$ is a minimally abnormal CLuN-model of $\Gamma^{G}$, that $\Delta=\left\{B \mid B \in \Gamma ; v_{M}(B)=1\right\}$, and that $v_{M}(A)=0$. $M$ is a CLuN-model of $\Delta$ that falsifies $A$. Hence, by Theorem 3.1, $\Delta \nvdash_{\mathbf{C L}} A$.

Theorem 4.5. If $\Gamma \cup\{A\} \subseteq \mathcal{W}^{\nsim}$, then $\Gamma \vdash_{\text {Strong }} A$ iff $\Gamma^{G} \vDash_{\text {ACLuN2 }} A$.

Proof. Let $\Gamma \cup\{A\} \subseteq \mathcal{W}^{\nsim}$. For the first direction, suppose that $\Gamma \vdash_{\text {Strong }}$ $A$. So, $\Delta \vdash_{\mathrm{CL}} A$ for all $\Delta \in M C S(\Gamma)$. By Lemma 4.13, $\{B \mid B \in$ $\left.\Gamma ; v_{M}(B)=1\right\} \in M C S(\Gamma)$ for all ACLuN2-models of $\Gamma^{G}$. Hence, by Lemma 4.14, all ACLuN2-models of $\Gamma^{G}$ verify $A$.

For the second direction, suppose that $\Gamma^{G} \vDash_{\text {ACLuN2 }} A$. So, all ACLuN2-models of $\Gamma^{G}$ verify $A$. Consider any $\Delta \in M C S(\Gamma)$. By Lemma 4.10, there is an ACLuN2-model $M$ of $\Gamma^{G}$ such that $\Delta=\{B \mid B \in \Gamma$; 
$\left.v_{M}(B)=1\right\}$. If $\Delta \nvdash_{\mathbf{C L}} A$, then, by Lemma 4.14, some ACLuN2-model $M$ of $\Gamma^{G}$ falsifies $A$, which is impossible. Hence, $\Delta \vdash_{\mathbf{C L}} A$ for all $\Delta \in$ $\operatorname{MCS}(\Gamma)$.

\subsection{C-based consequences - C-maximal normality}

At this point, I have to introduce a new strategy: C-Maximal Normality. As a general strategy applied to CLuN, it involves lots of complications. Fortunately, the present context allows for a simplified description. Remember first that we always consider the Guido-transformation $\Gamma^{G}$ of a set of premises $\Gamma$. Next, we know from Lemma 4.5 that, where $A \in \Gamma$, any CLuNmodel of $\Gamma^{G}$ verifies $A$ iff it falsifies $! \neg A$. However, the $\mathbf{C L u N - m o d e l s ~ w e ~}$ are interested in here are all minimally abnormal with respect to $\Gamma^{G}$, and for any such model $M, A \in A b(M)$ only if $\sim A \in \Gamma^{G}$ (see Lemma 4.11). Given this, a CLuN model $M$ of $\Gamma^{G}$ will be said to be a C-maximally normal CLuN-model of $\Gamma^{G}$ iff no CLuN-model $M^{\prime}$ of $\Gamma^{G}$ is such that $(\{\neg A \mid A \in \Gamma\}-A b(M))^{\#}>\left(\{\neg A \mid A \in \Gamma\}-A b\left(M^{\prime}\right)\right)^{\#}$ (where, as before,

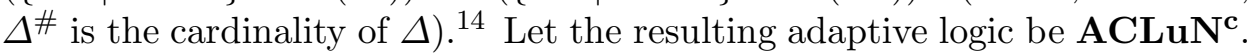

Theorem 4.6. If $\Gamma \cup\{A\} \subseteq \mathcal{W}^{\nsim}$, then $\Gamma \vDash_{C \text {-Based }} A$ iff $\Gamma^{G} \vDash_{\text {ACLuNc }^{c}} A$.

The proof is almost obvious in view of the proofs from the previous section. We first establish the analogues of the Lemmas from the previous section (except for Lemma 4.9), replacing $M C S(\Gamma)$ by $C M C S(\Gamma)$ and ACLuN2-model by $\mathbf{A C L u N} \mathbf{N}^{\mathbf{c}}$-model. The proof of the Theorem readily follows.

For the C-Based consequence relation, it makes a difference whether $\Gamma$ is a set or as a multiset. Similarly for the C-Maximal Normality strategy.

\subsection{Classical consequences - classicality}

The CL-consequence relation can be seen as a limit case within the context of the Rescher-Manor Mechanism. Indeed, we obviously have $\Gamma \vdash_{\mathbf{C L}} A$ iff $\Delta \vdash_{\mathbf{C L}} A$ for some $\Delta \subseteq \Gamma$. And, of course, this consequence relation is easily seen to be an inconsistency-'adaptive' logic obtained by applying an

\footnotetext{
${ }^{14}$ It is easily seen that all C-maximally normal $\mathbf{C L u N}$-models of $\Gamma^{G}$ are minimally abnormal CLuN-models of $\Gamma^{G}$. If $\Gamma$ is finite, the criterion is equivalent to $A b(M)^{\#}<$ $A b\left(M^{\prime}\right)^{\#}$ (which may be taken to define the C-Minimal Abnormality Strategy).
} 
extreme strategy to CLuN: select consistent models only - that is, the CLuN-models $M$ such that $v_{M}(\exists ! A)=0$ for all $A \cdot{ }^{15}$

\subsection{Weak consequences - normal selections}

The Weak Consequences and the Argued Consequences of $\Gamma$ cannot be characterized in terms of a unique selection of $\mathbf{C L u N - m o d e l s ~ o f ~} \Gamma^{G}$. Nevertheless, they have a nice abstract characterization and especially a nice dynamic proof theory. For both cases, I shall first devise a strategy. That these strategies were not discovered earlier is related to the fact that there is little use for anything richer than Minimal Abnormality in the context of the standard interpretation of a set of premises.

The inconsistency-adaptive logic obtained by applying the Normal Selections strategy to $\mathbf{C L u N}$ will be called $\mathbf{A C L u N}$ s. In the following Definitions and Lemma, $\Gamma \subseteq \mathcal{W}$.

Definition 4.19. A set $\Delta$ is normal with respect to $\Gamma$ iff $\Gamma \nvdash_{\mathbf{C L u N}} D E K(\Delta)$.

Definition 4.20. $\Gamma \vDash_{\text {ACLuNs }} A$ iff, for some $\Delta$ that is normal with respect to $\Gamma, A$ is verified by each CLuN-model $M$ of $\Gamma$ for which $A b(M) \cap \Delta=\emptyset$.

I shall rely on the following Lemma in Section 5. Its proof is obvious in view of the definitions.

Lemma 4.15. $\Gamma \vDash_{\text {ACLuNs }} A$ iff, for some $\Delta, \Gamma \vDash_{\mathbf{C L u N}} A \vee D E K(\Delta)$ whereas $\Gamma \nvdash_{\mathbf{C L u N}} \operatorname{DEK}(\Delta)$.

To simplify the following proof, let $\mathcal{M}_{\Delta}$ be the set of CLuN-models $M$ of $\Gamma^{G}$ for which $A b(M) \cap \Delta=\emptyset$.

TheOREM 4.7. If $\Gamma \cup\{A\} \subseteq \mathcal{W}^{\nsim}$, then $\Gamma \vdash_{\text {Weak }} A$ iff $\Gamma^{G} \vDash_{\text {ACLuNs }^{s}} A$.

Proof. Let $\Gamma \cup\{A\} \subseteq \mathcal{W}^{\nsim}$. For the first direction suppose that $\Gamma \vdash_{\text {Weak }} A$. In view of Definition 2.2, $\Delta \vdash_{\mathbf{C L}} A$ for some $\Delta \in M C S(\Gamma)$. By Theorem 3.1, $\Delta \vdash_{\text {CLuN }} A$. Hence, all CLuN-models of $\Gamma^{G}$ that verify $\Delta$, verify $A$. As $\Delta$ is $\neg$-consistent, some $\mathbf{C L u N}$-models of $\Gamma^{G}$ verify $\Delta$ by Lemma 4.4, and hence $\{\neg B \mid B \in \Delta\}$ is normal with respect to $\Gamma^{G}$. As $\Delta \subseteq \Gamma$, each CLuN-model $M$ of $\Gamma^{G}$ for which $A b(M) \cap\{\neg B \mid B \in \Delta\}=\emptyset$, falsifies $\neg B$ for all $B \in \Delta$, hence verifies $\Delta$, and hence verifies $A$.

\footnotetext{
${ }^{15}$ Each such model is equivalent to (verifies the same members of $\mathcal{W}$ as) some CLuNmodel such that $v(\sim A)=0$ for all $\sim A \in \sim^{\sim} \mathcal{W}^{+}$.
} 
For the second direction, suppose that, for some $\Delta$ that is normal with respect to $\Gamma^{G}$, all $M \in \mathcal{M}_{\Delta}$ verify $A$. By Definition $4.19, \mathcal{M}_{\Delta} \neq \emptyset$. Let $\Theta=\{B \mid \neg B \in \Delta ; B \in \Gamma\}$, and let $\Theta\urcorner=\{\neg B \mid B \in \Theta\}$. Clearly $\mathcal{M}_{\Delta} \subseteq \mathcal{M}_{\Theta}$. Hence, in view of Lemma 4.5, each $M \in \mathcal{M}_{\Delta}$ verifies $\Theta$. So, as $\mathcal{M}_{\Delta} \neq \emptyset$ and $\Theta \subseteq \Gamma, \Theta$ is $\neg$-consistent by Lemma 4.4. But then there is a $\Lambda$ such that $\Theta \subseteq \Lambda \in M C S(\Gamma)$.

Let $M$ be a minimally abnormal CLuN-model of $\Gamma^{G}$ that verifies $\Lambda$. As $\Theta \subseteq \Lambda, M$ verifies $\Theta$ and, by Lemma 4.12, $A b(M) \cap \Delta=\emptyset$. It follows that $M$ is a member of $\mathcal{M}_{\Delta}$, and hence that it verifies $A$. Hence $\Lambda \vdash_{\mathbf{C L}} A$ in view of Lemma's 4.13 and 4.14 .

\subsection{Argued consequences - two step}

Many Two Step strategies may be defined, but only one is relevant here. The inconsistency-adaptive logic obtained by applying the following Two Step strategy to $\mathbf{C L u N}$ will be called $\mathbf{A C L u N}{ }^{\mathrm{t}}$.

Definition 4.21. $\Gamma \vDash_{\text {ACLuN }^{\mathrm{t}}} A$ iff (i) for some $\Delta$ that is normal with respect to $\Gamma, A$ is verified by each $\mathbf{C L u N - m o d e l ~} M$ of $\Gamma$ for which $A b(M) \cap$ $\Delta=\emptyset$, and (ii) for all $\Delta$ that are normal with respect to $\Gamma, \neg A$ is falsified by some CLuN-model $M$ of $\Gamma$ for which $A b(M) \cap \Delta=\emptyset$.

The proof of the following two lemma's is obvious in view of this definition and the definitions in the previous Subsection.

Lemma 4.16. $\Gamma \vDash_{\text {ACLuNt }^{t}} A$ iff (i) for some $\Delta, \Gamma \vDash_{\mathrm{CLuN}} A \vee D E K(\Delta)$ and $\Gamma \nvdash_{\mathrm{CLuN}} D E K(\Delta)$, whereas (ii) for no $\Delta, \Gamma \vDash_{\mathrm{CLuN}} \neg A \vee D E K(\Delta)$ and $\Gamma \nvdash_{\mathbf{C L u N}} \operatorname{DEK}(\Delta)$.

Lemma 4.17. $\Gamma \vDash_{\text {ACLuN }^{t}} A$ iff $\Gamma \vDash_{\mathbf{A C L u N s}^{\mathrm{s}}} A$ and $\Gamma \nvdash_{\mathbf{A C L u N}^{\mathrm{s}}} \neg A$.

So, we shall be home by proving that the corresponding relation obtains between argued and weak consequences.

Lemma 4.18. Some $\Delta \subseteq \Gamma$ is an argument for $A$ iff $\Gamma \vdash_{W e a k} A$.

Proof. Suppose that $\Delta \subseteq \Gamma$ is an argument for $A$. By Definition $2.7 \Delta$ is consistent and $\Delta \vdash_{\mathbf{C L}} A$. Hence, $\Delta \subseteq \Theta$ for some $\Theta \in M C S(\Gamma)$. But then, by Definition 2.2 and the monotonicity of $\mathbf{C L}, A$ is a Weak consequence of $\Gamma$.

Suppose next that $\Gamma \vdash_{\text {Argued }} A$. By Definition 2.2, there is a $\Theta \in$ $\operatorname{MCS}(\Gamma)$ such that $\Theta \vdash_{\mathbf{C L}} A$. Let $\left\langle B_{1}, B_{2}, \ldots\right\rangle$ be a list of the members of $\Theta$; let $\Theta_{0}$ be $\Theta$; let $\Theta_{i+1}=\Theta_{i}-\left\{B_{i+1}\right\}$ iff $\Theta_{i}-\left\{B_{i+1}\right\} \vdash_{\mathbf{C L}} A$, and 


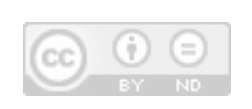

TOWARDS THE UNIFICATION ...

$\Theta_{i+1}=\Theta_{i}$ otherwise; finally, let $\Delta=\Theta_{1} \cap \Theta_{2} \cap \ldots$. Obviously, $\Delta$ is consistent, $\Delta \vdash_{\mathbf{C L}} A$, and, for no $B \in \Delta, \Delta-\{B\} \vdash_{\mathbf{C L}} A$. So, by Definition 2.7, $\Delta$ is an argument for $A$.

Lemma 4.19. $\Gamma \vdash_{\text {Argued }} A$ iff $\Gamma \vdash_{W e a k} A$ and $\Gamma \nvdash_{W e a k} \neg A$.

Proof. Obvious in view of Definition 2.8 and Lemma 4.18.

Theorem 4.8. If $\Gamma \cup\{A\} \subseteq \mathcal{W}^{\nsim}$, then $\Gamma \vdash_{\text {Argued }} A$ iff $\Gamma^{G} \vDash_{\text {ACLuN }^{\mathbf{t}}} A$.

Proof. Obvious in view of Lemmas 4.17 and 4.19, and Theorem 4.7.

\section{Dynamic proofs for the Rescher-Manor mechanism}

One of the main gains from the present results, is that the consequence relations of the Rescher-Manor Mechanism are provided with a (dynamic) proof theory. I shall present these proof theories in a unified manner and I spell out the conditions under which $A$ is finally derived in a proof from $\Gamma^{G}$. Space limitations force me to skip the metatheoretic proofs. This is a small loss: any logician that familiarizes himself or herself with [8] will see that they are obvious anyway.

Let me start with some general characteristics. Premise Rule: any $A \in$ $\Gamma^{G}$ may be written down at a line of the proof with the justification PREM and $\emptyset$ as its fifth element. To proceed faster, I now present two metarules. Unconditional Rule: if $\vdash_{\mathbf{C L u N}}\left(B_{1} \& \cdots \& B_{n}\right) \supset A$, and $B_{1}, \ldots, B_{n}$ occur in the proof with, respectively, the conditions $\Delta_{1}, \ldots, \Delta_{n}$, then one may add $A$ to the proof with the condition $\Delta_{1} \cup \cdots \cup \Delta_{n}$. Conditional Rule: if $\vdash_{\mathrm{CLuN}} \operatorname{DEK}\left(C_{1}, \ldots, C_{m}\right) \vee\left(\left(B_{1} \& \cdots \& B_{n}\right) \supset A\right)$, and $B_{1}, \ldots, B_{n}$ occur in the proof with, respectively, the conditions $\Delta_{1}, \ldots, \Delta_{n}$, then one may add $A$ to the proof with the condition $\left\{C_{1}, \ldots, C_{m}\right\} \cup \Delta_{1} \cup \cdots \cup \Delta_{n}$.

Any consequence relation will have its specific Marking Rule and/or Deletion Rule. A marked line is considered as OUT; the wff occurring on it (its second element) is not considered as derived. Yet, a marked line may be used to derive further lines, and may be used to decide whether another line should be marked. A deleted line is also considered as OUT but provides no useful information for deriving further lines or for marking lines.

As appears from the above Theorems, an inconsistency-adaptive consequence $A$ of $\Gamma^{G}$ is only a consequence of $\Gamma$ according to some consequence 
relation from the Rescher-Manor Mechanism iff $A$ is finally derived in the proof and is a member of $\mathcal{W}^{\nsim} .16$

For Free consequences, we appeal to the Reliability strategy. The Marking Rule is as follows: if $D E K(\Delta)$, but not $D E K(\Theta)$ for any $\Theta \subset \Delta$, has been derived unconditionally, mark the lines the fifth element of which contains a member of $\Delta$. $A$ is finally derived in a proof from $\Gamma^{G}$ iff $A$ occurs as the second element of an unmarked line $i$ of the proof and, if $i$ is marked in an extension of the proof, then it is unmarked in a further extension.

The Strong consequence relation requires the Minimal Abnormality strategy. The Marking Rule is (complex and) as described in Section 3.3. $A$ is finally derived in a proof from $\Gamma^{G}$ iff $A$ occurs as the second element of an unmarked line $i$ of the proof and, if $i$ is marked in a (possibly infinite) extension of the proof, then it is unmarked in a further extension.

The $C$-Based consequence relation relies on the C-Maximal Normality strategy. Let $\Gamma\urcorner=\{\neg A \mid A \in \Gamma\}$. Marking most easily proceeds in two steps. First, all lines are marked as for the Minimal Abnormality strategy. Of the remaining lines, a line is marked iff its fifth element contains less members of $\Gamma^{\urcorner}$than the fifth element of another unmarked line. ${ }^{17}$ The conditions for final derivability are as for the Minimal Abnormality strategy.

The Classical consequence relation requires a strategy that was labelled Classicality before, but is, in the present context, better characterized as Blindness: never delete any line (do not see that the premises falsify its condition). Anything derived at a stage is finally derived.

The Weak consequence relation appeals to the Normal Selections strategy. There is no marking rule but a deletion rule: delete any line that has $\Delta$ as its fifth element if $\operatorname{DEK}(\Delta)$ has been unconditionally derived. Anything deleted at a stage is finally deleted. So, $A$ is finally derived at a stage iff it is not deleted in any extension of the proof.

Finally, we come to the Argued consequence relation. The Deletion Rule is as for Weak consequences. The Marking Rule is as follows: iff $A$ is derived at line $i$ and $\neg A$ at line $j$, mark both lines. These instructions should be taken literally. A marked line $i$ may be used to derive a further line. Whether the latter should be marked depends directly on the Marking Rule. That line $i$ is marked does not entail that lines derived from it should also be

\footnotetext{
${ }^{16}$ For those concerned: it is possible to restrict the proof to members of $\mathcal{W}^{\nsim}$. For example, the Premise Rule may be replaced by: if $\sim \neg A \in \Gamma^{G}, A$ may be written down at a line of the proof with the justification PREM and $\{\neg A\}$ as its fifth element.

${ }^{17}$ If $A$ is derived at line $i$ with condition $\Delta$, it can also be derived at a line $j$ with condition $\Delta \cup \Theta$ (for any $\Theta \subseteq \Gamma^{\urcorner}$). Of course, for some $\Theta$, line $j$ will be marked.
} 


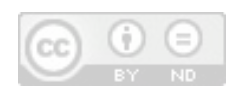

TOWARDS THE UNIFICATION ...

marked. For example, if no premise is an Argued Consequence of $\Gamma$, then some (or even all) premises may have (CL-)consequences that are Argued Consequences of $\Gamma$. Example: if $\Gamma=\{p \& q,(p \supset \neg q) \& r\}$, then no premise is an argued consequence of $\Gamma$, but $p, q$, and $r$ are.

Anything deleted at a stage is finally deleted. $A$ is finally derived at a stage iff $A$ occurs unmarked at that stage, is not deleted in any extension of the proof and, if it is marked in any (finite) extension of the proof, then it is unmarked in a further extension (because $\neg A$ or, where $A=\neg B, B$ is deleted in the further extension).

\section{In conclusion}

The unification of inconsistency-handling mechanisms is the first (and obvious) gain from the present results. All popular 'flat' inconsistency-handling mechanisms have been shown to be inconsistency-adaptive logics. So, the latter offer a solid base for studying existing variants and devising new ones. This does not mean that the Rescher-Manor Mechanism should be given up. Quite to the contrary: it presents an outlook that is sufficiently different from the adaptive logic approach to warrant that back and forth translations will (for some time) be fruitful. The recapture of the Rescher-Manor Mechanism in terms of inconsistency-adaptive logics offers the possibility to devise sensible further consequence relations within both.

An extremely important gain is that the consequence relations defined within the Rescher-Manor Mechanism are now provided with a dynamic proof theory. There are two, quite different, aspects to this. The first is that the Rescher-Manor Mechanism is connected to our everyday thinking. The latter does not proceed in terms of $M C S$, but in terms of deriving consequences that later (as our understanding of the premises grows) may be given up. The second is related to undecidable cases. Here the original formulation of the Rescher-Manor Mechanism breaks down, but the dynamic proofs are still effective. One may (dynamically) derive consequences from the premises, and take decisions based on one's present best insights. Such decisions may have to be revised later, but at the time, are justified in terms of those present best insights. The ensuing decisions are not infallible, but they are the best that can be taken according to any sensible (contemporary) view on human rationality.

The Rescher-Manor Mechanism is moreover provided with a semantics (in terms of $\mathbf{C L u N - m o d e l s ) . ~ T h e ~ i m p o r t a n c e ~ o f ~ t h i s ~ i s ~ m o s t ~ e a s i l y ~ s e e n ~}$ if confronted with the claim, in [14, p. 20], that "In the presence of in- 
consistency, the approaches developed in [the Rescher-Manor Mechanism] must be syntactic in nature, since they explicitly use formulas that appear in the belief base originally, while two inconsistent belief bases are semantically equivalent (in a trivial way)." Moreover, there is a nice semantics that captures the dynamics of the proofs. ${ }^{18}$

The present unification is asymmetric. It is easily seen from the previous sections that the inconsistency-adaptive logics that define the RescherManor Mechanism are very special cases. Many other adaptive logics, and even many other inconsistency-adaptive logics, are available.

Actually, other adaptive logics have quite interesting application contexts in which the consequence relations defined within the Rescher-Manor Mechanism are not suitable. For one thing, the latter presuppose that each premise derives from a single source. Often, however, sources cannot be retraced. For example, a knowledge system of an individual or group does not contain information on the sources of most of its items. And even if the sources may be retraced, we often want to retain parts of the information provided by sources of which most information is rejected. The Argued Consequences partly take care of this, but it is obvious that other inconsistency-adaptive logics offer more possibilities in this respect. More important than these theoretical arguments are arguments provided from historical case studies. In this connection, I refer to [16] and [18].

The most important difference between the Rescher-Manor Mechanism and adaptive logics applied to premises under the standard interpretation, is that the negations are interpreted classically in the former case, and paraconsistently in the latter. The former offer a consistent interpretation of the premises (with Weak Consequences as the sole exception) whereas the latter offer an inconsistent interpretation. The latter is superior in cases where inconsistencies have to be resolved in view of non-logical means. They locate the inconsistencies (and possibly reveal their connections). Actually, the cases where inconsistencies have to be resolved by non-logical means abound. Only seldom the inconsistent premises contain themselves sufficient information to devise their consistent improvement. This holds for inconsistent mathematical theories as well as for inconsistent empirical theories. Consistent set theories were not arrived at from the Strong, Argued, or C-Based consequences of Frege's (let alone Cantor's) set theory. Similarly for, for example, Clausius' theory in thermodynamics (see [16]). The

\footnotetext{
18 The reader may verify that the results of [6] are independent of the specific strategy employed.
} 
same a fortiori applies for everyday theories and for knowledge systems of individuals or groups. Summarizing: many consequence relations defined within the Rescher-Manor Mechanism offer a consistent interpretation of the inconsistent premises on so-called logical grounds. Whether this interpretation is suitable in a specific context, however, is a non-logical question. Adaptive logics based on the standard interpretation of the premises offer an inconsistent interpretation of the premises and hence do not prejudge on the consistent improvement of an inconsistent theory (or set of theories). ${ }^{19}$

The integration of the Rescher-Manor Mechanism within the adaptive logic family deserves careful further study. The insights from the latter will lead to further adaptive logics and to a better understanding of the suitability of adaptive logics to specific contexts. Only along this road, we shall be able to arrive at a better understanding of inconsistency-handling mechanisms and, indirectly, of the nature of logic. ${ }^{20}$

\section{References}

[1] Diderik Batens. Paraconsistent extensional propositional logics. Logique et Analyse, 90-91:195-234, 1980.

[2] Diderik Batens. Dynamic dialectical logics as a tool to deal with and partly eliminate unexpected inconsistencies. In J. Hintikka and F. Vandamme, editors, The Logic of Discovery and the Logic of Discourse, pages 263-271. Plenum Press, New York, 1985.

[3] Diderik Batens. Dialectical dynamics within formal logics. Logique et Analyse, 114:161-173, 1986.

[4] Diderik Batens. Static and dynamic paraconsistent logics and their use in expert systems. $C C-A I, 3: 33-50,1986$.

[5] Diderik Batens. Dynamic dialectical logics. In Graham Priest, Richard Routley, and Jean Norman, editors, Paraconsistent Logic. Essays on the Inconsistent, pages 187-217. Philosophia Verlag, München, 1989.

[6] Diderik Batens. Blocks. The clue to dynamic aspects of logic. Logique et Analyse, 150-152:285-328, 1995. Appeared 1997.

[7] Diderik Batens. Dynamic semantics applied to inconsistency-adaptive logics. In Logical Investigations, volume 5, pages 74-85. Moscow, "NAUKA", 1998.

\footnotetext{
19 As appears from [24], the latter may be the result of disambiguating certain nonlogical terms.

${ }^{20}$ Unpublished papers in the reference section (and many others) are available from the internet address http://logica.rug.ac.be/centrum/writings/.
} 
[8] Diderik Batens. Inconsistency-adaptive logics. In Ewa Orłowska, editor, Logic at Work. Essays Dedicated to the Memory of Helena Rasiowa, pages 445-472. Physica Verlag (Springer), Heidelberg, New York, 1999.

[9] Diderik Batens. Minimally abnormal models in some adaptive logics. Synthese, 125:5-18, 2000.

[10] Diderik Batens. A survey of inconsistency-adaptive logics. In Batens et al. [13], pages 49-73.

[11] Diderik Batens and Joke Meheus. A tableau method for inconsistency-adaptive logics. In Roy Dyckhoff, editor, Automated Reasoning with Analytic Tableaux and Related Methods, Lecture Notes in Artificial Intelligence Vol. 1847, pages 127-142. Springer, 2000.

[12] Diderik Batens and Joke Meheus. Shortcuts and dynamic marking in the tableau method for adaptive logics. Studia Logica, 69:221-248, 2001.

[13] Diderik Batens, Chris Mortensen, Graham Priest, and Jean Paul Van Bendegem, editors. Frontiers of Paraconsistent Logic. Research Studies Press, Baldock, UK, 2000.

[14] Salem Benferhat, Didier Dubois, and Henri Prade. Some syntactic approaches to the handling of inconsistent knowledge bases: A comparative study. Part 1: The flat case. Studia Logica, 58:17-45, 1997.

[15] Kristof De Clercq. Two new strategies for inconsistency-adaptive logics. Logic and Logical Philosophy, in print.

[16] Joke Meheus. Adaptive logic in scientific discovery: the case of Clausius. Logique et Analyse, 143-144:359-389, 1993. Appeared 1996.

[17] Joke Meheus. An extremely rich paraconsistent logic and the adaptive logic based on it. In Batens et al. [13], pages 189-201.

[18] Joke Meheus. Inconsistencies in scientific discovery. Clausius's remarkable derivation of Carnot's theorem. In Geert Van Paemel and et al., editors, Acta of the XXth International Congress of History of Science. Brepols, in print.

[19] Graham Priest. Minimally inconsistent LP. Studia Logica, 50:321-331, 1991.

[20] Nicholas Rescher. Hypothetical Reasoning. North-Holland, Amsterdam, 1964.

[21] Nicholas Rescher. The Coherence Theory of Truth. Clarendon, Oxford, 1973.

[22] Nicholas Rescher and Robert Brandom. The Logic of Inconsistency. A study in Non-Standard Possible-World Semantics and Ontology. Blackwell, Oxford, 1980.

[23] Nicholas Rescher and Ruth Manor. On inference from inconsistent premises. Theory and Decision, 1:179-217, 1970. 
[24] Guido Vanackere. Ambiguity-adaptive logic. Logique et Analyse, 159:261-280, 1997. Appeared 1999.

[25] Guido Vanackere. HL2. An inconsistency-adaptive and inconsistency-resolving logic for general statements that might have exceptions. Journal of Applied Non-Classical Logics, 10:317-338, 2000.

[26] Guido Vanackere. Preferences as inconsistency-resolvers: the inconsistencyadaptive logic PRL. Logic and Logical Philosophy, in print.

[27] Timothy Vermeir. Inconsistency-adaptive arithmetic. To appear.

\section{DiDERIK BATENS}

Centre for Logic and Philosophy of Science

Universiteit Gent, Belgium

Diderik.Batens@rug.ac.be 